\title{
Modeling resonant trojan motions in planetary systems
}

\author{
Christos Efthymiopoulos ${ }^{1}$ and Rocío I. Páez ${ }^{2}$ \\ ${ }^{1}$ Research Center for Astronomy and Applied Mathematics, Academy of Athens, Greece \\ ${ }^{2}$ Dip. di Matematica, Universitá di Roma "Tor Vergata", Italy \\ emails: cefthim@academyofathens.gr, paez@mat.uniroma2.it
}

\begin{abstract}
We consider the dynamics of a small trojan companion of a hypothetical giant exoplanet under the secular perturbations of additional planets. By a suitable choice of actionangle variables, the problem is amenable to the study of the slow modulation, induced by secular perturbations, to the dynamics of an otherwise called 'basic' Hamiltonian model of two degrees of freedom (planar case). We present this Hamiltonian decomposition, which implies that the slow chaotic diffusion at resonances is best described by the paradigm of modulational diffusion.
\end{abstract}

Keywords. celestial mechanics, methods: analytical, chaotic diffusion

\section{Introduction}

Despite extensive search, no pairs of co-orbital exoplanets have been discovered so far. Some reasons for the unlikeliness of the co-orbital configuration are discussed in Giuppone et al. (2012), Haghighipour (2013), Dobrovolskis (2013) and Pierens and Raymond (2014). Dynamical obstructions appear in the formation process as well as during the migration and/or capture of the planets into resonance. Besides these constraints, however, there is also the question of the long-term stability of co-orbital motions. This means the stability of the orbits over timescales comparable to the age of the hosting system.

In a recent work (Páez and Efthymiopoulos 2014) we initiated a study of the long-term stability in a hypothetical configuration in which a small (considered massless) planet moves around the Lagrangian points of a giant primary. Numerical simulations have shown that up to Earth-sized trojan planets can appear close to gaseous giants (Beaugé et al. 2007, Lyra et al. 2009). This dynamical system is a case of the elliptic restricted three body problem (ERTBP), or, with additional planets, the 'restricted multi-body problem' (RMBP). Alternative applications of the RMBP encompass co-orbital satellites of a planet, asteroids, and artificial trojan objects in a Sun-planet or planet-moon system.

In an accompanying poster (Páez and Efthymiopoulos, this volume) we outline one of our so-far obtained numerical results, referring to the diffusion timescales in the case of initial conditions taken close to some so-called secondary resonances within the co-orbital domain. Several authors (e.g. Érdi et al. 2007, 2009, Schwarz et al. 2007) have stressed the importance of secondary resonances in the problem of long-term stability. Related numerical works, applied to Jupiter's trojan asteroids, are Marzari et al. (2003), Robutel and Gabern (2006), Robutel and Bodossian (2009). Our own numerical work compares maps of the resonant structure, as depicted in a suitably defined domain of action variables (i.e. proper elements), with maps of the stability times for initial conditions within the resonance web. We found evidence of a tight correlation between the two maps (see Páez and Efthymiopoulos, this volume). 
In the sequel we briefly discuss how our Hamiltonian formulation in action-angle variables is introduced in the framework of the RMBP, as well as the consequences this formulation leads to regarding the dynamical characterization of the problem.

\section{Summary of the Hamiltonian formulation}

A summary of our formulation is the following: assuming all perturbing planets far from mean motion resonances, by a suitable sequence of canonical transformations we arrive (in the planar case) at expressing the Hamiltonian of the RMBP as:

$$
H=H_{b}\left(J_{s}, \phi_{s}, Y_{f}, \phi_{f}, Y_{p} ; e_{0}\right)+H_{s e c}\left(J_{s}, \phi_{s}, Y_{f}, \phi_{f}, Y_{p}, \phi, P_{1}, \phi_{1}, \ldots, P_{S}, \phi_{S}\right) .
$$

i) The pairs $\left(Y_{f}, \phi_{f}\right),\left(J_{s}, \phi_{s}\right),\left(Y_{p}, \phi\right)$ are action-angle conjugate variables corresponding to the 'short-period', 'synodic' and 'secular' motions of the trojan body respectively. The short-period terms correspond physically to epicyclic oscillations. The synodic oscillations describe the 'long period' librations around the Lagrangian points L4 or L5. The action variable $J_{s}$ determines the value of the 'proper libration' (see Milani (1993), or Beaugé and Roig (2001) for the definition of trojan proper elements). The action $Y_{p}$ labels the 'proper eccentricity'. The angle $\phi$ measures phase oscillations around an angle $\beta$ (see below) which expresses the relative difference between the arguments of perihelia of the trojan body and the giant primary. We note that an analysis omitted here allows to see that the form of the Hamiltonian (2.1) implies that the oscillations of $\beta$ are bounded. Finally, the pairs $\left(\phi_{i}, P_{i}\right), i=1, \ldots, s$ are action-angle variables for the oscillations of the eccentricity vectors of the $S$ additional planets.

ii) We call the first term $H_{b}$ in (2.1) the 'basic model'. The angle $\phi$ is ignorable in $H_{b}$, implying that $Y_{p}$ is a constant of motion under the dynamics of $H_{b}$ alone. The parameter $e_{0}$ is the mean modulus of the eccentricity vector of the giant primary. Thus, $H_{b}$ represents a system of two degrees of freedom, wherein both $e_{0}$ and $Y_{p}$ act as parameters, i.e. the 'forced' $\left(e_{0}\right)$ and 'proper' $\left(e_{p}=\sqrt{-2 Y_{p}}\right)$ eccentricity.

iii) The term $H_{\text {sec }}$ contains only trigonometric terms depending on the slowly varying angles $\phi, \phi_{i}, i=1, \ldots, s$. Hence, $H_{\text {sec }}$ introduces only secular perturbations to the dynamics under $H_{b}$. In particular, $H_{s e c}$ causes a slow pulsation of the chaotic separatrixlike layers at the borders of the resonances arising under $H_{b}$. As shown in Páez and Efthymiopoulos 2014, this phenomenon is best described by the paradigm of 'modulational diffusion' (Chirikov et al. 1985).

iv) The form of the function $H_{b}$ is identical in the ERTBP and the RMBP, setting $e_{0}=e^{\prime}$ and $\beta=\omega$ in the former, where $e^{\prime}$ is the (constant) eccentricity of the primary, and $\omega^{\prime}=0$ its pericentric position. This formal equivalence implies that the qualitative features of the diffusion along resonances, as they appear in the plane of the action variables $J_{s}, Y_{p}$, are similar in the RMPP and the ERTBP. Examples of the latter are studied in Páez and Efthymiopoulos (2014).

We now summarize the derivation of the Hamiltonian (2.1). We assume that, far from mean-motion resonances, the time evolution of the eccentricity vectors of all massive bodies can be approximated by quasi-periodic formulae

$$
\begin{aligned}
& e^{\prime} \exp i \omega^{\prime}=e_{0}^{\prime} \exp i\left(\omega_{0}^{\prime}+g^{\prime} t\right)+\sum_{k=1}^{s} A_{k} \exp i\left(\omega_{k 0}^{\prime}+g_{k} t\right) \\
& e_{j} \exp i \omega_{j}=B_{j 0} \exp i\left(\omega_{0 j}+g^{\prime} t\right)+\sum_{k=1}^{s} B_{k j} \exp i\left(\omega_{k j}^{\prime}+g_{k} t\right)
\end{aligned}
$$

setting, without loss of generality, $\omega_{0}^{\prime}=0$. The constants $g^{\prime}$, and $g_{j}, j=1, \ldots s$ are 
secular frequencies associated with the primary and the $S$ planets respectively. Also, we assume that the condition $e_{0}^{\prime}>\sum_{k=1}^{S} A_{k}$ holds for the giant primary, implying an average constant rate of precession of its perihelion with frequency $g^{\prime}$. One has $e^{\prime}=e_{0}^{\prime}+F$, $\omega^{\prime}=\phi^{\prime}+G$, where $\phi^{\prime}=g^{\prime} t$ and $F$ and $G$ are of first order in the amplitudes $A_{k}$, $k=1, \ldots, s$. Averaged over the mean longitudes $\lambda_{1}, \ldots, \lambda_{S}$ the Hamiltonian reads

$$
H=-\frac{1}{2(1+x)^{2}}+I_{3}+g^{\prime} I^{\prime}+\sum_{j=1}^{S} g_{j} I_{j}-\mu R\left(\lambda, \omega, x, y, \lambda^{\prime}, \phi^{\prime} ; e_{0}^{\prime}\right)-\mu R_{2}-\sum_{j=1}^{S} \mu_{j} \mathcal{R}_{j}
$$

where: i) $x=\sqrt{a}-1, y=\sqrt{a}\left(\sqrt{1-e^{2}}-1\right)$ are Delaunay action variables, $(a, e)$ being the major semi-axis and eccentricity of the trojan body (in units in which $a^{\prime}=1$ for the primary), and $(\lambda, \omega)$ the mean longitude and argument of the perihelion. The variables $I_{3}, I^{\prime}, I_{j}, j=1, \ldots, S$ are dummy actions congugate to the angles $\lambda^{\prime}, \phi^{\prime}=g^{\prime} t$ and $\phi_{j}=g_{j} t$. ii) $R$ is has the same form as the disturbing function in the ERTBP with the substitution $e_{0} \rightarrow e^{\prime}, \phi^{\prime} \rightarrow \omega^{\prime}$, with $\mu$ equal to the primary's mass parameter (all functions and variables are considered in the heliocentric frame). iii) $R_{2}$, expressing the indirect effects of the $S$ additional planets, comes from replacing $e^{\prime}=e_{0}^{\prime}+F\left(\phi^{\prime}, \phi_{j}\right)$, $\omega^{\prime}=\phi^{\prime}+G\left(\phi^{\prime}, \phi_{j}\right)$ in the disturbing function of the ERTBP and Taylor-expanding around $e_{0}^{\prime}$ and $\phi^{\prime}$, assuming $F$ and $G$ small quantities. $\mathcal{R}$ is of degree one or higher in the mass parameters $\mu_{j}, j=1, \ldots, S$. iv) Finally, $\mathcal{R}_{j}$ are the (averaged over mean longitudes) direct terms of the $S$ additional planets.

The canonical transformation $\tau=\lambda-\lambda^{\prime}, \beta=\omega-\phi^{\prime}, J_{3}=I_{3}+x, P^{\prime}=I^{\prime}+y$ allows to re-express the hamiltonian in terms of the resonant angle $\tau$ and the relative argument of pericenter difference $\beta$. The Hamiltonian can be recast as $H=\langle H\rangle+H_{1}$, where

$$
\begin{aligned}
\langle H\rangle= & -\frac{1}{2(1+x)^{2}}-x+J_{3}-g^{\prime} y-\mu\langle R\rangle\left(\tau, \beta, x, y ; e_{0}^{\prime}\right) \\
H_{1}= & g^{\prime} P^{\prime}+\sum_{j=1}^{S} g_{j} I_{j}-\mu \tilde{R}\left(\tau, \beta, x, y, \lambda^{\prime}, \phi^{\prime} ; e_{0}^{\prime}\right) \\
& -\sum_{j=1}^{S} \mu_{j} R_{j}\left(x, y, \beta, \phi^{\prime}, \phi_{1}, \ldots, \phi_{s}\right)-\mu \mathcal{R}_{2}\left(x, y, \tau, \beta, \phi^{\prime}, \phi_{1}, \ldots, \phi_{s}\right)
\end{aligned}
$$

with $\langle R\rangle=\frac{1}{2 \pi} \int_{0}^{2 \pi} R d \lambda^{\prime}, \tilde{R}=R-\langle R\rangle$. The Hamiltonian $\langle H\rangle$ allows to determine the forced equilibrium by the solution to the system of equations $\partial\langle H\rangle / \partial x=\partial\langle H\rangle / \partial y=$ $\partial\langle H\rangle / \partial \tau=\partial\langle H\rangle / \partial \beta=0$. One finds that

$$
\left(\tau_{0}, \beta_{0}, x_{0}, y_{0}\right)=\left(\pi / 3, \pi / 3,0, \sqrt{1-e_{0}^{\prime 2}}-1\right)+O\left(g^{\prime}\right) .
$$

Note that the forced equilibrium represents a relative configuration, i.e., the eccentricity vector of the trojan body has the same modulus $e_{0}$ and a constant relative angle with respect to the mean eccentricity vector of the primary. This result follows also by a careful inspection of the formulae provided in Morais (2001).

Expanding around the forced equilibrium, we introduce new variables

$$
\begin{gathered}
v=x-x_{0}, \quad u=\tau-\tau_{0}, \quad Y=-\left(W^{2}+V^{2}\right) / 2, \quad \phi=\arctan (V / W) \\
V=\sqrt{-2 y} \sin \beta-\sqrt{-2 y_{0}} \sin \beta_{0}, \quad W=\sqrt{-2 y} \cos \beta-\sqrt{-2 y_{0}} \cos \beta_{0} .
\end{gathered}
$$

The variables $(v, u)$ describe the motion in the synodic plane, while the action variable $Y$ measures the distance of an orbit from the forced equilibrium position in the secular 
plane $(\sqrt{-2 y} \cos \beta, \sqrt{-2 y} \sin \beta)$. Finally, we introduce the canonical transformations $Y_{p}=$ $Y+J_{3}, \phi_{f}=\lambda^{\prime}-\phi$, and

$$
J_{s}=\frac{1}{2 \pi} \int_{C}\left(v-v_{0}\right) d\left(u-u_{0}\right)
$$

where the integration is over a closed invariant curve $C$ around $\left(u_{0}, v_{0}\right)$, with conjugate angle $\phi_{s}$. Substituting these transformations yields the form (2.1) of the Hamiltonian.

The study of the basic model allows to identify the most important secondary resonances, which are commensurabilities between the fast and synodic frequencies $\omega_{f}=\dot{\phi}_{f}$, $\omega_{s}=\dot{\phi}_{s}$. The fast frequency is related to the secular frequency $g=\dot{\phi}$ by $\omega_{f}=1-g$, in units in which the mean motion of the giant primary is equal to 1 . The general form of a resonance is

$$
m_{f} \omega_{f}+m_{s} \omega_{s}+m g+m^{\prime} g^{\prime}+m_{1} g_{1}+\ldots+m_{S} g_{S}=0
$$

with $m_{f}, m_{s}, m, m^{\prime}, m_{j}$ (with $j=1, \ldots, S$ ) integers. The resonances of the basic model exist in the complete hierarchy of problems, from the planar circular restricted three body problem $\left(s=0, g^{\prime}=0, e_{0}^{\prime}=0\right)$ up to the complete multi-body problem. For the mass parameters of giant exoplanets the most important resonances are of the form $\omega_{f}-n \omega_{s}=0$, with $n$ in the range $4 \leqslant n \leqslant 12$ for typical mass parameters of the gaseous primary. In the frequency space $\left(\omega_{f}, \omega_{s}, g\right)$, these resonances define planes normal to the plane $\left(\omega_{f}, \omega_{s}\right)$ which intersect each other along the $g$-axis. All other resonances with $|m|+\left|m^{\prime}\right|+\left|m_{1}\right|+\ldots+\left|m_{S}\right|>0$ intersect transversally one or more planes of the main resonances. We refer to such resonances as 'transverse' if $\left|m_{f}\right|+|n|>0$, or 'secular' if $\left|m_{f}\right|+|n|=0$. In Efthymiopoulos and Páez (2014), we show that the diffusion along transverse or secular resonances is of the Arnold type, hence very slow. On the other hand, there are transverse resonances which accumulate to multiplets around the main ones, thus producing a faster (modulational) diffusion.

\section{Acknowledgments}

R. I. P. was supported by the Astronet-II Training Network (PITN-GA-2011-289240). C. E. was supported by the Research Committee of the Academy of Athens (Grant 200/815) and by an IAU Symposium Grant.

\section{References}

Beaugé, C. \& Roig, F. 2001, Icarus 153, 391-415.

Chirikov, B. V., Lieberman, M. A., Shepelyansky, D. L., \& Vivaldi, F. M. 1985, Physica D 14, 289-304.

Dobrovolskis, A. 2013, Icarus 226, 1636-1641.

Érdi B., Nagy I, Sándor Zs. \& Süli, A., Fröhlich G. 2007, MNRAS 381, 33-40.

Érdi, B., Forgács-Dajka, E., Nagy, I., \& Rajnai, R. 2009, Cel. Mech. Dyn. Astron. 104, 145-158

Giuppone, C. A., Benítez-Llambay, P., \& Beaugé, C. 2012, MNRAS 421, 356-368.

Haghighipour, N., Capen, S., \& Hinse, T. 2013, Cel. Mech. Dyn. Astron. 117, 75-89.

Lyra, W., Johansen, A., Klahr, H., \& Piskunov, N. 2009, A\&A 493, 1125-1139.

Marzari, F., Tricarino, P. \& Scholl, H. 2003, MNRAS 345, 1091-1100.

Milani, A. 1993, Cel. Mech. Dyn. Astron. 57, 59-94.

Morais, M. H. M. 2001, A\&\&A 369, 677-689.

Páez, R.I. \& Efthymiopoulos, C. 2014, Cel. Mech. Dyn. Astron. (in press), arXiV 1410.1407.

Pierens, A. \& Raymond, S. N. 2014, MNRAS 442, 2296-2303.

Robutel, P. \& Gabern, F. 2006, MNRAS 372, 1463-1482.

Robutel, P. \& Bodossian, J. 2009, MNRAS 399, 69-87.

Schwarz, R., Dvorak, R., Süli, A., \& Érdi B. 2007 A $\& A$ 474, 1023-11029. 